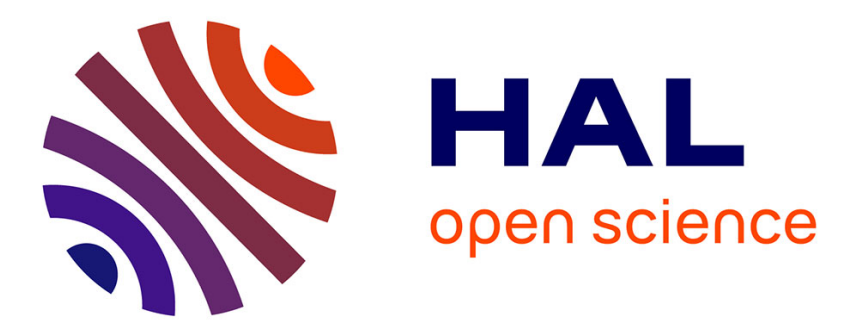

\title{
Shape Gradient for Isogeometric Structural Design
}

\author{
Louis Blanchard, Régis Duvigneau, Anh-Vu Vuong, Bernd Simeon
}

\section{To cite this version:}

Louis Blanchard, Régis Duvigneau, Anh-Vu Vuong, Bernd Simeon. Shape Gradient for Isogeometric Structural Design. Journal of Optimization Theory and Applications, 2014, 161 (2), pp.361-367. 10.1007/s10957-013-0435-0 . hal-00998594

\section{HAL Id: hal-00998594 \\ https://hal.inria.fr/hal-00998594}

Submitted on 2 Jun 2014

HAL is a multi-disciplinary open access archive for the deposit and dissemination of scientific research documents, whether they are published or not. The documents may come from teaching and research institutions in France or abroad, or from public or private research centers.
L'archive ouverte pluridisciplinaire HAL, est destinée au dépôt et à la diffusion de documents scientifiques de niveau recherche, publiés ou non, émanant des établissements d'enseignement et de recherche français ou étrangers, des laboratoires publics ou privés. 


\title{
Shape Gradient for Isogeometric Structural Design
}

\author{
Louis Blanchard • Régis Duvigneau • \\ Anh-Vu Vuong · Bernd Simeon
}

Received: date / Accepted: date

\begin{abstract}
The transfer of geometrical data from CAD (Computer Aided Design) to FEA (Finite-Element Analysis) is a bottleneck of automated design optimization procedures, yielding a loss of accuracy and cumbersome software couplings. Isogeometric analysis methods propose a new paradigm, that allows one to overcome these difficulties by using a unique geometrical representation, yielding a direct relationship between geometry and analysis. In this study, in the framework of linear elasticity problems, we investigate its use for sensitivity analysis and, more specifically, shape gradient computations.
\end{abstract}

Keywords Sensitivity analysis · Linear elasticity

Mathematics Subject Classification (2000) 49Q12 $\cdot 74 \mathrm{~B} 05$

L. Blanchard and R. Duvigneau

INRIA OPALE Project-Team, 2004 route des lucioles 06902 Sophia-Antipolis, France

E-mail: Regis.Duvigneau@inria.fr

A.-V. Vuong and B. Simeon

Tech. Universität Kaiserslautern, Paul-Ehrlich-Strasse 31, 67663 Kaiserslautern, Germany 


\section{Introduction}

Multi-disciplinary optimization procedures are nowadays commonly used by engineers to solve complex problems. However, the processing of the geometrical data through the design loop is still a delicate issue: several different representations of the geometry coexist and several conversions are required, yielding additional overhead and extra difficulties from the theoretical and practical point of view. Usually, the geometry of the engineering system of interest is defined using CAD (Computer Aided Design) software, on the basis of high-order representations. NURBS (Non-Uniform Rational B-Spline) basis functions are considered as standard in this context [1]. Cubic functions are often used, exhibiting a $C^{2}$ regularity. The predominant analysis tools used to solve PDEs, such as FEA (Finite-Element Analysis) software, rely on a grid to describe the computational domain, which defines the geometry with piecewise linear functions and $C^{0}$ regularity only. It is remarkable that some errors are introduced before the beginning of any physical analysis. In the context of design optimization, some inconsistencies occur, since the performance estimation is based on the approximate geometry, whereas the optimizer updates the high-order NURBS representation. The same inconsistencies hold for gradient estimates, if a gradient-based optimization method is employed. The isogeometric analysis approach, proposed recently by Hughes et. al. [2], promises to resolve, or at least alleviate, these issues. Therefore, this study is devoted to the estimation of shape derivatives in this particular context and the application to optimization problems ruled by linear elasticity equations. 


\section{Isogeometric Analysis in Linear Elasticity}

We consider an open domain $\Omega \subset \mathbb{R}^{d}$, which represents a deformable solid subject to external forces $\mathbf{g}$, in $d=2$ or $d=3$ dimensions. Its boundary is composed of three disjoint parts, $\partial \Omega=\Gamma_{N} \cup \Gamma_{D} \cup \Gamma$ with $\Gamma \cap \Gamma_{D}=\varnothing$, $\Gamma \cap \Gamma_{N}=\varnothing$ and $\Gamma_{N} \cap \Gamma_{D}=\varnothing$. Dirichlet and Neumann boundary conditions are prescribed, respectively, on $\Gamma_{D}$ and $\Gamma_{N}$, whereas $\Gamma$ is considered as optimization variable (moving boundary). The governing equations of linear elasticity describe the displacement field $\mathbf{u}$ as solution of the PDE system:

$$
\left\{\begin{array}{rlrlrl}
-\operatorname{div} \sigma(\mathbf{u})= & 0 & \text { in } \Omega, & \mathbf{u}=0 & \text { on } \Gamma_{D}, \\
\sigma(\mathbf{u}) \cdot \mathbf{n}=\mathbf{g} & \text { on } \Gamma_{N}, & \sigma(\mathbf{u}) \cdot \mathbf{n}=0 & \text { on } \Gamma,
\end{array}\right.
$$

where $\mathbf{n}$ is the outward unit normal vector and $\sigma(\mathbf{u})$ the second-order stress tensor, defined by Hooke's law. The variational formulation of Eq. (1) is the starting point for isogeometric analysis. The weak form reads as follows: Find the physical displacement field $\mathbf{u} \in \mathcal{V}=\left\{\varphi \in H^{1}(\Omega)^{d}, \varphi=0\right.$ on $\left.\Gamma_{D}\right\}$ such that:

$$
\int_{\Omega}[2 \mu \epsilon(\mathbf{u}): \epsilon(\mathbf{v})+\lambda \operatorname{div} \mathbf{u} \operatorname{div} \mathbf{v}] d \Omega=\int_{\Gamma_{N}} \mathbf{g} \cdot \mathbf{v} d \Gamma \quad \forall \mathbf{v} \in \mathcal{V}
$$

Isogeometric analysis [2] is aimed at a better integration of FEA and CAD methods, by using a unique representation basis for the geometry and the discrete solution fields. It proposes to discretize the computational domain exactly, by using a NURBS basis originating from CAD. NURBS basis functions are defined in a parametric domain $\Omega_{0}$. They can be represented in the physical domain $\Omega$ by introducing the transformation $F: \Omega_{0} \rightarrow \Omega, \boldsymbol{\xi} \mapsto F(\boldsymbol{\xi})=\mathbf{x}(\boldsymbol{\xi})$. 
E.g., for $d=2$, any point of coordinates $\mathbf{x}=(x, y)^{T}$ in the physical domain $\Omega$ is mapped to a point of parameters $\boldsymbol{\xi}=(\xi, \eta),{ }^{T}$ in the parametric domain $\Omega_{0}$. Two- or three-dimensional NURBS basis functions are defined as the bivariate or trivariate tensor product of one-dimensional basis functions. The latter have compact supports and are constructed using the so-called knot vector $\Xi=\left(\xi_{0}, \ldots, \xi_{l}\right) \in \mathbb{R}^{l+1}$, with $l=n+p+1$, where $p$ is the degree of the functions and $n$ the number of functions considered [1]. NURBS basis functions are rational extensions of B-Spline basis functions $N_{i, p} i=0, \cdots, n$, that are computed recursively by $N_{i, 0}(\xi)=1$, if $\xi_{i} \leq \xi<\xi_{i+1}, 0$ otherwise, and:

$$
N_{i, p}(\xi)=\frac{\xi-\xi_{i}}{\xi_{i+p}-\xi_{i}} N_{i, p-1}(\xi)+\frac{\xi_{i+p+1}-\xi}{\xi_{i+p+1}-\xi_{i+1}} N_{i+1, p-1}(\xi) .
$$

Note that the quotient $0 / 0$ is defined as zero. The transformation of the parametric domain $\Omega_{0}$ to the physical domain $\Omega$ is defined by associating a control point to each basis function. E.g., for $d=2$, we have:

$$
\mathbf{x}(\xi, \eta)=\sum_{i \in \mathcal{I}} \sum_{j \in \mathcal{J}} N_{i}(\xi) N_{j}(\eta) \mathbf{X}_{i j},
$$

where $\mathbf{X}_{i j} \in \mathbb{R}^{2}$ represents the coordinates of the control point of indices $(i, j)$ in the physical domain. Using such a representation, the domain $\Omega$ is described as a single B-Spline patch, without approximation by a piecewise linear grid.

In the isogeometric paradigm, the same representation is employed for both the geometry and the physical fields. Therefore, the discretized displacement field $\mathbf{u}_{h}$ is constructed as linear combination of the B-Splines that define the geometry:

$$
\mathbf{u}_{h}(\mathbf{x})=\sum_{i \in \mathcal{I}} \sum_{j \in \mathcal{J}} N_{i}(\xi) N_{j}(\eta) \mathbf{U}_{i j}=\sum_{i \in \mathcal{I}} \sum_{j \in \mathcal{J}} \hat{N}_{i j}(\mathbf{x}) \mathbf{U}_{i j},
$$


where the basis functions $\hat{N}_{i j}(\mathbf{x})$ are defined by $\hat{N}_{i j} \circ F(\xi, \eta)=N_{i}(\xi) N_{j}(\eta)$. Consequently, the restriction of the variational formulation leads to a system of linear equations $\mathbf{K} \mathbf{U}=\mathbf{F}$ :

$$
\begin{aligned}
& K_{i j, k l}=\int_{\Omega}\left[2 \mu \epsilon\left(\hat{N}_{i j}\right) \cdot \epsilon\left(\hat{N}_{k l}\right)+\lambda \operatorname{div}\left(\hat{N}_{i j}\right) \operatorname{div}\left(\hat{N}_{k l}\right)\right] d \Omega, \\
& F_{k l}=\int_{\Gamma_{N}} \mathbf{g} \cdot \hat{N}_{k l} d \Gamma .
\end{aligned}
$$

The entries of the stiffness matrix, and also those of the force vector $\mathbf{F}$, are computed by applying classical quadrature rules in the parametric domain, in accordance with the evaluation process of the B-Spline functions in Eq. (3).

\section{Shape Gradient Computation}

As optimization problem, we consider the minimization of the compliance functional. To be well posed, i.e. admit at least one solution, this problem requires some smoothness or geometrical constraints [3]. Therefore, $\beta>0$ being a positive Lagrange multiplier, we consider the minimization problem:

$$
\min _{\Omega \in \mathcal{U}_{a d}} L(\Omega, \beta), \quad L(\Omega, \beta)=\int_{\Gamma_{N}} \mathbf{g} \cdot \mathbf{u} d \Gamma+\beta\left(\int_{\Omega} d \Omega-V_{0}\right),
$$

where $V_{0}$ is the initial volume and $\mathcal{U}_{a d}=\left\{\Omega \subset \mathbb{R}^{d}, \int_{\Omega} d \Omega=V_{0}\right\}$.

In order to solve the problem in Eq. (7) using a gradient-based method, we introduce the concept of shape derivative [4], in the context of isogeometric approximations. The theoretical foundations of this concept can be found in [5] and applications in [6]. Let $\mathbf{v}: \mathbb{R}^{d} \rightarrow \mathbb{R}^{d}$ denote a sufficiently smooth admissible vector field, that defines the shape deformation due to the optimization process. By differentiating Eq. (7), under the assumptions that $\mathbf{g}, \mathbf{u} \in H^{2}(\Omega)^{d}$, 
it can be shown that the shape derivative of the Lagrangian, for a given shape deformation $\mathbf{v}$, is:

$$
d L(\Omega, \beta ; V)=\int_{\Gamma}\left(\beta-\left(2 \mu|\epsilon(\mathbf{u})|^{2}+\lambda|\operatorname{div} \mathbf{u}|^{2}\right)\right) \mathbf{v} \cdot \mathbf{n} d \Gamma .
$$

Therefore, a possible choice to ensure a compliance decrease [3] is to consider a shape deformation defined by $\mathbf{v}=\left(2 \mu|\epsilon(\mathbf{u})|^{2}+\lambda|\operatorname{div} \mathbf{u}|^{2}-\beta\right) \mathbf{n}$ on $\Gamma$, and 0 on $\Gamma_{D} \cup \Gamma_{N}$, where $\beta$ is chosen to satisfy the constraint. It is convenient to extend this definition on the whole domain $\Omega$, in order to update the geometry as a whole. Finally, the deformation field is defined by solving a second elasticity problem:

$$
\left\{\begin{aligned}
-\operatorname{div} \sigma(\mathbf{v}) & =0 & & \text { in } \Omega, \\
\mathbf{v} & =0 & & \text { on } \Gamma_{D} \cup \Gamma_{N}, \\
\sigma(\mathbf{v}) \cdot \mathbf{n} & =\left(2 \mu|\epsilon(\mathbf{u})|^{2}+\lambda|\operatorname{div} \mathbf{u}|^{2}-\beta\right) & & \text { on } \Gamma .
\end{aligned}\right.
$$

One can notice that, in Eq. (9), we do not impose the deformation field $\mathbf{v}$ on the boundary $\Gamma$ as a Dirichlet condition. Instead, we consider a Neumann condition for $\mathbf{v}$. Indeed, the use of such a Neumann boundary condition enables to increase the regularity of the deformation field $\mathbf{v}$, which may suffer from a loss of regularity [3]. This approach ensures the shape change to be a descent direction [3]. Concluding, the problem defined by Eq. (9) is solved by using exactly the same approach as for the linear elasticity problem given by Eq. (1). In particular, an isogeometric approximation is employed, yielding a deformation of the domain in terms of control point displacements. The shape optimization procedure can be summarized by the following algorithm:

1. Initialization of the domain defined by the control points $\mathbf{X}^{0}$ and $k=0$ 
2. Start of optimization loop

3. Solve system (6) yielding the structural displacement $\mathbf{U}^{k}$

4. Solve system (9) yielding the shape deformation $\mathbf{V}^{k}$

5. Initialization of step length $t^{k}$

6. Compute the multiplier $\beta^{k}$ such that $\mathbf{X}^{k+1}=\mathbf{X}^{k}+t^{k} \mathbf{V}^{k}$ is admissible

7. Solve system (6) yielding the structural displacement $\mathbf{U}^{k+1}$

8. Compute the compliance for $\left(\mathbf{X}^{k+1}, \mathbf{U}^{k+1}\right)$

9. If Goldstein-Armijo criteria not fulfilled, $t^{k}$ is updated and goto (6)

10. If converged then stop, else $k \leftarrow k+1$ and goto (3)

11. End of optimization loop

The determination of the Lagrange multiplier $\beta^{k}$ for each domain update is achieved by solving an internal one-parameter problem. Details regarding the procedure can be found in [7]. We underline the fact that, according to the isogeometric analysis paradigm, a unique basis is used to represent the geometry of the domain, the structural displacement field and the deformation field for the optimization.

\section{An Application}

The proposed methodology is applied to a $3 \mathrm{D}$ problem. The objective is to optimize the shape of an open-spanner, as illustrated by Fig. 1. Two problems are considered successively: in a first optimization exercise, only the top and bottom boundaries are considered as optimization variables, whereas in a second exercise, the shape of the lateral boundaries is also optimized. The 
objective is the reduction of the compliance, subject to a constant volume constraint. Additional constraints are also imposed to maintain the shape of the open-spanner symmetric with respect to horizontal and vertical planes.

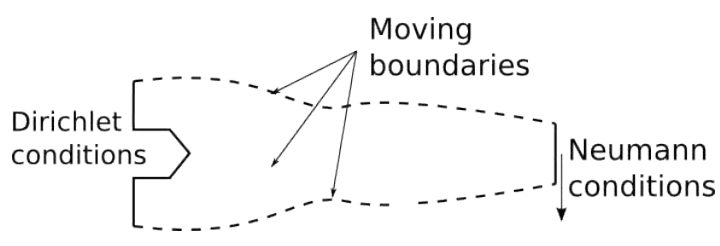

Fig. 1 Description of the open-spanner test-case.

The domain is parameterized using linear basis functions in the vertical and crosswise directions, and quadratic functions in the lengthwise direction. The net of control points counts $6 \times 7 \times 2$ points, respectively, in horizontal, vertical and crosswise directions. Thus, the two optimization exercises account for 48 and 192 variables. The evolution of the cost function during the optimization procedure is displayed in Fig. 2. As seen, convergence is achieved in a few iterations and a very low computational time. A comparison of initial and final displacement fields is depicted in Fig. 3. The optimization procedure yields an enlargement of the left part of the open-spanner, whereas the right part becomes thinner to fulfill the constant volume constraint. For the second exercise, the open-spanner is made thinner in the crosswise direction, which enables to enlarge it significantly in the left part. 


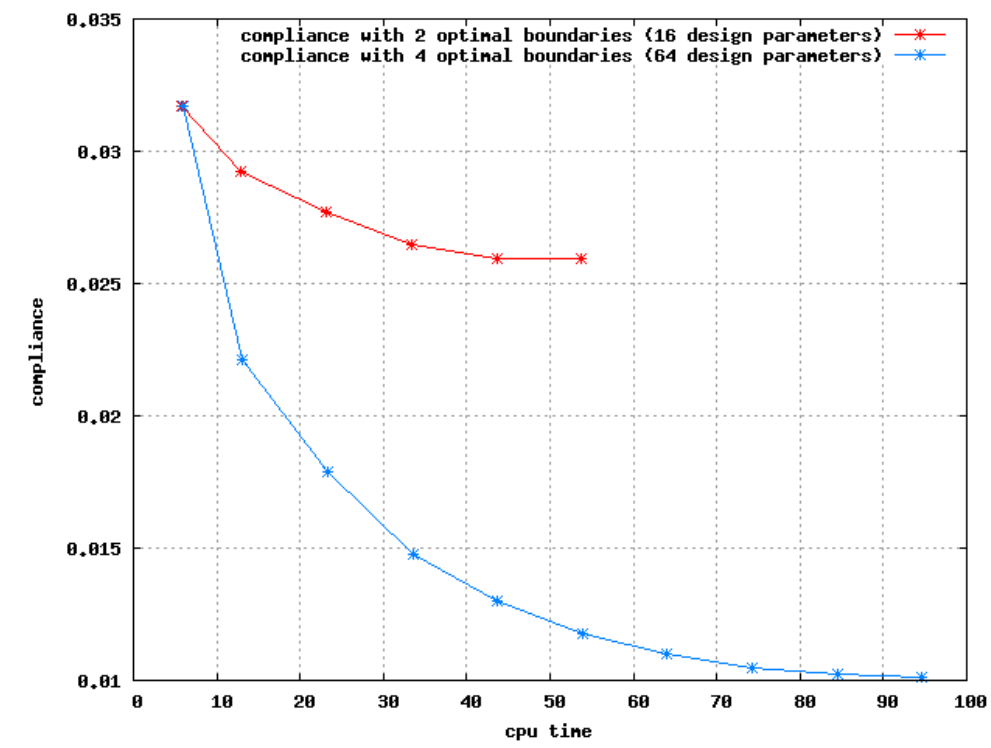

Fig. 2 Cost function evolution for the two optimization exercises with respect to CPU time.
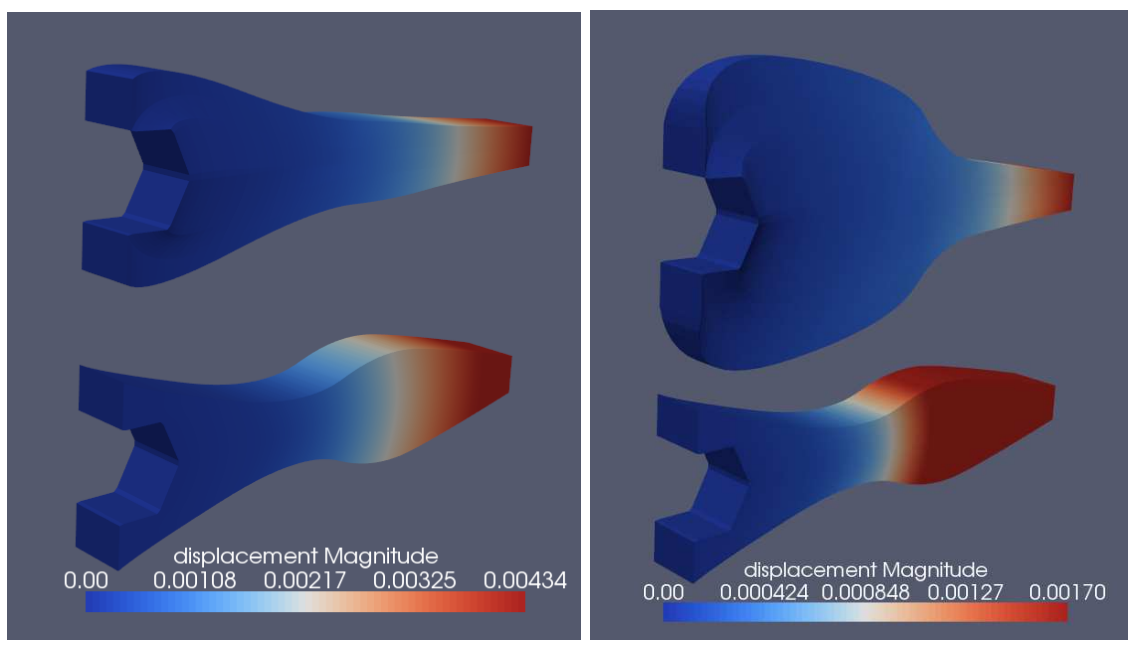

Fig. 3 Comparison of the initial (bottom) and final (top) displacement fields for the first (left) and second (right) exercises.

\section{Conclusions}

This study has demonstrated that isogeometric analysis provides an attractive theoretical and practical framework for sensitivity analysis and shape 
derivative computations. The concept of shape derivatives has been set-up in this context, for linear elasticity problems, and used for practical shape optimization exercises. Although the current approach has been restricted to the minimization of the compliance, it could be extended to other functionals by solving the corresponding adjoint problem.

Nevertheless, several improvements are still required to face industrial problems: from a practical point of view, the approach needs to be extended to multi-patch representations. Moreover, local refinement techniques should be introduced to improve computational efficiency. In this context, the major challenges are the control of the regularity at the patch boundaries and the linear independency of the refined basis functions [8].

Acknowledgements This study was supported by the European Union within the Projects 218536 "EXCITING" and 284981 "TERRIFIC" ( 7th Framework Program).

\section{References}

1. Sabin, M.: Cad system components. Computer Aided Design 25(3), 119-140 (1993)

2. Hughes, T., Cottrell, J., Bazilevs, Y.: Isogeometric analysis: Cad, finite elements, nurbs, exact geometry, and mesh refinement. Computer Methods in Applied Mechanics and Engineering (194), 4135-4195 (2005)

3. Allaire, G., Jouve, F., Toader, A.: Structural optimization using shape sensitivity analysis and a level-set method. J. Comput. Physics 194 (2004)

4. Sokolowski, J., Zolésio, J.P.: Introduction to shape optimization. Springer Verlag (1992)

5. Delfour, M., Zolesio, J.P.: Shapes and geometries: analysis, differential calculus, and optimization. SIAM (2001) 
6. Azegami, H., Fukumoto, S., Aoyam, T.: Shape optimization of continua using nurbs as basis functions. Structural and Multidisciplinary Optimization (47), 247-258 (2013)

7. Blanchard, L., Duvigneau, R., Vuong, A.-V., Simeon, B.: Shape Gradient Computation in Isogeometric Analysis for Linear Elasticity. INRIA Research Report RR-8111 (2012)

8. Vuong, A., Giannelli, C., Juttler, B., Simeon, B.: A hierarchical approach to adaptive local refinement in isogeometric analysis. Computer Method in Applied Mechanics and Engineering 200(49-52) (2011) 